\title{
Optical transfer function of an acousto-optic heterodyning image processor
}

\author{
T. C. Poon and A. Korpel \\ Department of Electrical and Computer Engineering, University of Iowa, Iowa City, Iowa 52242
}

Received March 23, 1979; revised manuscript received June 11, 1979

\begin{abstract}
Image processing by means of an interactive pupil technique is described. A Bragg-diffraction sound cell is used
\end{abstract} to achieve both wave-front division and temporal-carrier offset.

In a conventional incoherent scanning or imaging system, limitations exist on image processing because of the constraints on magnitude and phase of the optical transfer function (OTF). ${ }^{1}$ It has been pointed out that such limitations may be overcome by using a two-pupil image-forming system.,3 For a particular class of such systems using the so-called pupil-interaction method, ${ }^{2}$ the OTF is characterized by the cross correlation of two-pupil transmission functions rather than by the autocorrelation of a single function, as in conventional methods. ${ }^{4}$

The implementation of such two-pupil systems usually involves creating a distinction between the pupils on the basis of either spatial ${ }^{5,6}$ or temporal ${ }^{7}$ carriers. This makes it possible to select the desired signal (or image) and separate it from other undesired components. The two pupils themselves are created by either amplitude ${ }^{2,7}$ or wave front ${ }^{3,5}$ division. A synthetichologram method has been described. ${ }^{8}$

Rhodes ${ }^{9,10}$ recently described temporal-carrier, amplitude-division systems using a Mach-Zehnder interferometer. A time-varying phase shift is introduced in one of the interferometer arms by means of either a constant-velocity mirror or a vibrating mirror. Difference-frequency signals are detected by nonintegrating detectors and further processed by various phase-demodulator techniques.

In this Letter we report on a simple pupil-interaction processing technique in which both carrier-frequency generation and wave-front division ${ }^{3}$ are brought about by acousto-optic Bragg diffraction. The system uses coherent light, and its output (a heterodyne current) is characterized by amplitude and phase, which generally is characteristic of coherent systems. The system, however, is incoherent in the sense that its operation is described by the same formalism as that used for incoherent pupil-interaction methods.

An idealized and highly schematic version of the system is shown in Fig. 1. (For the sake of simplicity, the exact entrance angle of the input beam is not shown, nor have we indicated the true shape of the beams as they propagate through the system.) A He-Ne laser L directs a collimated beam of light onto a sound cell $\mathrm{S}$ operating in the Bragg regime. ${ }^{11}$ The relevant output of the cell consists of two identical beams, differing in frequency by $\Delta \nu$ and in direction of propagation by $\alpha$ $\simeq(\lambda / V) \Delta \nu, V$ being the velocity of sound and $\lambda$ the vacuum wavelength of the light. The frequency $\Delta y$ is equal either to the sound frequency itself or to the difference between two sound frequencies that are present simultaneously. We will return to this later.

Lens $L_{1}$ forms, in its back focal plane; the Fourier transform $U_{1}^{\prime}, V_{1}{ }^{\prime}$ of the fields $U_{0}, V_{0}$ describing the two beams in the sound cell. A phase and amplitude transparency $\Gamma_{1}$ acts on $U_{1}{ }^{\prime}, V_{1}{ }^{\prime}$ to create $U_{1}, V_{1}$, after which a second lens $\mathrm{L}_{2}$ forms Fourier transforms $U_{2}, V_{2}$ in its back focal plane. The total field in plane 2, which is the filtered image of the field $U_{0}+V_{0}$, is used to scan or sample a transparency $\Gamma_{2}(x, y)$. By scanning or sampling is meant that successive points $(x, y$, in transparency coordinates) of $\Gamma_{2}$ are brought into coincidence with the center $\left(x_{2}, y_{2}=0\right)$ of the field in plane 2. This most easily accomplished by moving the transparency through the field. Photodiode PD collects all the light and delivers an rf heterodyne current at frequency $\Delta \nu$, which may be described by the phasor $I(x, y)$. The phase and amplitude of the photodiode current, as a function of $x$ and $y$, constitutes the scanned and processed version of the transparency $\Gamma_{2}(x, y)$. Using the optical convention for the phasor $A=$ $|A| \exp (-j \phi)$;

$$
|A| \cos (2 \pi \nu t+\phi)=\operatorname{Re}[A \exp (-j 2 \pi \nu t)],
$$

we write the two fields entering plane 2 ,

$$
\begin{aligned}
& U_{2}\left(x_{2}, y_{2}\right) \exp \left(-j \frac{2 \pi \Delta \nu}{2} t\right) \\
& \text { and } V_{2}\left(x_{2}, y_{2}\right) \exp \left(+j \frac{2 \pi \Delta \nu}{2} t\right),
\end{aligned}
$$

so that, apart from inessential constants, we have:

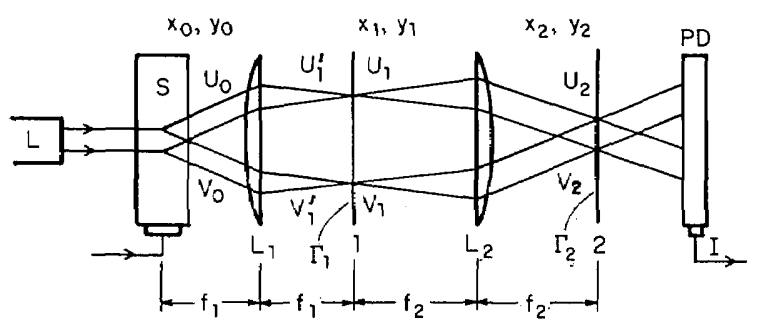

Fig. 1. Idealized processor 


$$
\begin{array}{r}
I(x, y)=\iint U_{2}\left(x_{2}, y_{2}\right) V_{2} *\left(x_{2}, y_{2}\right) \\
\times\left|\Gamma_{2}\left(x+x_{2}, y+y_{2}\right)\right|^{2} \mathrm{~d} x_{2} \mathrm{~d} y_{2},
\end{array}
$$

where it has been assumed that the transparency $\Gamma_{2}$ is not frequency sensitive. Equation (2) may be written:

$$
I(-x,-y)=\left(U_{2} V_{2}^{*}\right) \circledast\left|\Gamma_{2}\right|^{2},
$$

where $\circledast$ denotes correlation. From Eq. (3) it follows that

$$
\mathscr{F}\{I\}=\mathscr{F}^{*}\left\{I^{*}(-x,-y)\right\}=\mathcal{F}^{*}\left\{U_{2} * V_{2}\right\} \mathfrak{F}\left\{\left|\Gamma_{2}\right|^{2}\right\},
$$

where $\mathcal{F}$ denotes the Fourier-transform operation, i.e.,

$$
\mathscr{F}\{I\}\left(f_{x}, f_{y}\right)=\iint I(x, y) \exp \left(-j 2 \pi f_{x} x-j 2 \pi f_{y} y\right) \mathrm{d} x \mathrm{~d} y .
$$

The OTF of the scanning system is defined as

$$
\mathrm{OTF}=\mathscr{F}\{I\} \mid / \mathscr{F}\left\{\left|\Gamma_{2}\right|^{2}\right\}=\mathscr{F}^{*}\left\{U_{2} * V_{2}\right\} \text {. }
$$

Note that $I$ is a complex quantity; hence the modulus of the OTF is not necessarily symmetric. We will return to this later.

The fields $U_{2}, V_{2}$ are related to $U_{1}$ and $V_{1}$ by

$$
\begin{aligned}
& U_{2}\left(x_{2}, y_{2}\right)=\mathscr{F}\left\{U_{1}\left(\frac{x_{1}}{\lambda f_{2}}, \frac{y_{1}}{\lambda f_{2}}\right)\right\}, \\
& V_{2}\left(x_{2}, y_{2}\right)=\mathcal{F}\left\{V_{1}\left(\frac{x_{1}}{\lambda f_{2}}, \frac{y_{1}}{\lambda f_{2}}\right)\right\} .
\end{aligned}
$$

Substituting Eqs. (8) and (7) into Eq. (6) we find, after some manipulation,

$$
\mathrm{OTF}=U_{1} \circledast V_{1} \text {, }
$$

by which is meant

$$
\begin{aligned}
\operatorname{OTF}\left(f_{x}, f_{y}\right) & =\iint U_{1}\left(x_{1}, y_{1}\right) \\
& \times V_{1}^{*}\left(x_{1}-\lambda f_{2} f_{x}, y_{1}-\lambda f_{2} f_{y}\right) \mathrm{d} x_{1} \mathrm{~d} y_{1} .
\end{aligned}
$$

As mentioned before, the functions $U_{1}$ and $V_{1}$ are the transformed beams originating from a sound cell and modified by the transparency $\Gamma_{1}$. For large-enough beam width and frequency difference $\Delta \nu$, the beams are well separated in plane 1 , making it possible to modify each one individually. In our experiment we first demonstrate the simplest case, i.e., $\Gamma_{1}=1$. Figure 2(a) shows the functions $U_{1}$ and $V_{1}$ (for simplicity we use rectangles) separated by a distance $s$. The OTF, which is real in this case, is shown in Fig. 2(b). It is clearly a bandpass response, limited however to positive spatial frequencies. The latter feature does not represent a
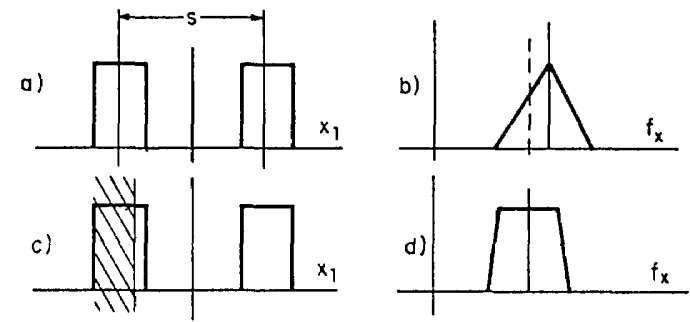

Fig. 2. (a) Separate beams in plane 1. (b) Resulting $|\mathrm{OTF}|$ is a band-pass filter. (c) One beam partially blocked. (d) Resulting $|\mathrm{OTF}|$ shifted to lower frequencies. limitation because the transparency $\left|\Gamma_{2}\right|^{2}$ to be processed is real, and hence the negative spatial frequencies are redundant. The asymmetry of the OTF with respect to $f_{x}$ corresponds to an impulse-response function $\left[I(x)\right.$ for $\left.\Gamma_{2}\left(x_{2}\right)=\delta\left(x+x_{2}\right)\right]$ that is complex and, as may be seen readily, of the form

$$
B(x) \exp \left(-j 2 \pi \frac{x s}{\lambda f_{2}}\right),
$$

where $B(x)$ is real.

The factor $\left(s / \lambda f_{2}\right)^{-1}$ in the exponent of Eq. (11) may be recognized as the fringe separation in plane 2 . The fringes in question are of course the image of the fringes in the sound cell, magnified by a factor $M=f_{2} / f_{1}$. They move in plane 2 with the appropriate velocity so as to generate a frequency $\Delta \nu$ when intercepted by a narrow slit, such as is used to measure the impulse response $I(x)$. Moving the slit in the $X_{2}$ direction causes the signal to shift in phase, as predicted by Eq. (11).

It is interesting that this feature makes it possible directly to transfer the phase modulation of a spatial carrier recorded on $\Gamma_{2}$ onto the current $I$ (or, inversely, to record a phase-modulated carrier) with a spot size too coarse to resolve this carrier (unfortunately, the required angular aperture is not reduced; rather, the lens is not completely filled).

In the second part of our experiment we show, in a qualitative way, that a true cross correlation is involved, by blocking part of $U_{1}$ with a knife edge, as shown in Fig. 2(c). The bandpass filter should now move to a lower frequency [Fig. 2(d)], the shift in center frequency increasing as more of $U_{1}$ is blocked.

Our experimental setup is a practical implementation of Fig. 1, using, for example, telescopes to broaden the Gaussian beam and cylinder lenses to tailor the beam to fit the sound cell. The input to the sound cell consisted of a $57-\mathrm{MHz}$ carrier, $100 \%$ amplitude modulated at $4 \mathrm{MHz}$ and thus creating equal amplitude components at 53 and $61 \mathrm{MHz}$. We used the two beams generated by these components rather than the zero- and first-order because the lower mixing frequency at 8 $\mathrm{MHz}$ is easier to process. The experiment is essentially one-dimensional in that the actual optical configuration used collapsed the image of the sound cell in plane 2 to a line along the $X_{2}$ axis. In what follows we have left out many quantitative details, such as beam size, because such data would be meaningless without an exhaustive description of the actual optical configuration. Details of the latter are not pertinent to the principle of operation, which is all we want to confirm in our experiments. A complete and detailed analysis will be published later.

To determine the impulse response of the scanner, we moved a narrow slit (much narrower than the fringe spacing) across plane 2 and measured phase and amplitude of the $8-\mathrm{MHz}$ current from the photodiode. A typical curve with $\Gamma_{1}=1$ is shown in Fig. 3(a).

In order to find the modulus of the OTF, we positioned a Ronchi ruling in plane 2. Rotating the ruling changes the effective spatial frequency in the relevant direction (i.e., perpendicular to the running fringes). Figure 3(b) shows the magnitude of the OTF so measured for difference frequencies of 4 and $8 \mathrm{MHz}$, re- 


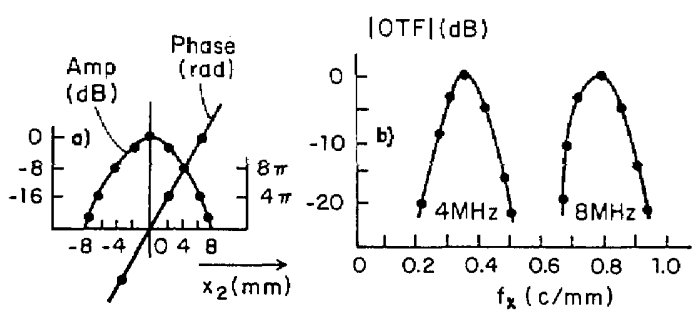

Fig. 3. (a) Phase and amplitude of impulse response for $\Gamma_{1}$ $=1$ and $\Delta \nu=8 \mathrm{MHz}$. (b) Corresponding OTF at $\Delta \nu=8 \mathrm{MHz}$ and $4 \mathrm{MHZ}$.

spectively. Note that changing $\Delta \nu$ effectively changes $s$ in Fig. 2(a). The 8-MHz curve corresponds to a large value of $s$ and hence to a higher center frequency of the bandpass filter. (Note that, strictly speaking, the experiment ought to be performed with a sinusoidal grating; it may be shown, however, that, with our experimental parameters, the error is very small if a Ronchi ruling is used.)

In the second experiment (at $4 \mathrm{MHz}$ ) we partially blocked one of the beams in plane 1 until the output current decreased by some arbitrary amount $(7 \mathrm{~dB})$ and then measured the (normalized) $|\mathrm{OTF}|$ again. Figure 4 (a) shows that the bandpass filter has indeed moved to lower frequencies.

As a further check we measured the shift in center frequency versus the degree of blocking, i.e., versus the amount of current reduction. The result is shown in Fig. 4(b), which qualitatively confirms the cross-correlation aspect of the $\mathrm{OTF}$.

It should be remarked that the technique is highly sensitive to the presence of dust, scratches, or other imperfections on any of the elements following the sound cell. This is, of course, in the nature of the method because any such imperfection acts as an additional mask $\Gamma$, modifying the response or causing a spurious signal. For this reason it is necessary that the photodiode be placed in an image plane of the separated beams; this eliminates some of the spurious signals generated by the nonuniform response of the diode surface.
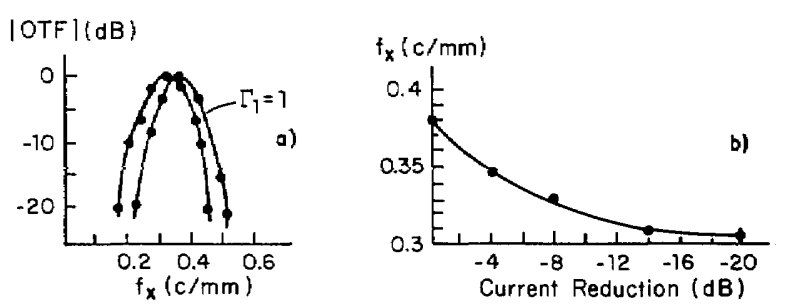

Fig. 4. (a) $|O T F|$ 's for $\Gamma=1$ and $\Gamma=$ knife edge, as in 2 (c). (b) Shift in bandpass center as a function of current reduction through blocking.

As a final note, we point out the possibility of creating a chirp-type impulse response (phase a quadratic function of $x$ ) in an out-of-focus plane near plane 2 by making $U_{1}$ a delta function. This carries obvious implications to holographic recording, analogous to synthetic-aperture radar. We plan to investigate this further in the near future.

\section{References}

1. W. Lukosz, J. Opt. Soc. Am. 52, 827 (1962).

2. A. W. Lohmann and W. T. Rhodes, Appl. Opt. 17, 1141 (1978).

3. D. Goerlitz and F. Lanzl, Opt. Commun. 20, 68 (1977).

4. J. W. Goodman, Introduction to Fourier Optics (McGraw-Hill, New York, 1968).

5. A. W. Lohmann, Appl. Opt. 16, 261 (1977).

6. W. Stoner, Appl. Opt. 17, 2454 (1978).

7. W. T. Rhodes, Appl. Opt. 16, 265 (1977).

8. P. Chavel and S. Lowenthal, J. Opt. Soc. Am. 66, 14 (1976).

9. W. T. Rhodes, in Proceedings of the 1978 International Optical Computing Conference (Institute of Electrical and Electronics Engineers, New York, 1979).

10. W. T. Rhodes, in Proceedings of the Electro-Optics/Laser 1978 Conference (Industrial and Scientific Conference Management, Chicago, Ill., 1979).

11. A. Korpel, "Acousto-optics," in Applied Solid State Science, Vol. 3, R. Wolfe, ed. (Academic, New York, 1972). 